\title{
Minidosis de ácido acetil salicílico como tratamiento profiláctico de la enfermedad hipertensiva del embarazo
}

\author{
Fernando Hernández*; María Fernanda Martínez **; Adriana Camero L.***; Juan Alberto Pinzón R. ****
}

\begin{abstract}
RESUMEN: EI presente estudio evalúa si el ácido acetil salicílico ingerido a bajas dosis (100 mg./día) a partir del segundo trimestre hasta la semana 38 de gestación previene la hipertensión inducida por el embarazo en las pacientes con alto riesgo de presentarla. Analizamos una población total de 97 pacientes, divididas en tres subgrupos de riesgo: primigestantes, pacientes con antecedente de hipertensión inducida por el embarazo y pacientes con hipertensión crónica mediante un estudio controlado, aleatorio, prospectivo, doble ciego entre el primero de Junio de 1991 y Octubre de 1995 en el Hospital Militar Central. No se observaron evidencias estadísticamente significativas que demuestren la existencia de un beneficio en cuanto al suministro indiscriminado del ASA en los grupos de riesgo evaluados para la prevención de la hipertensión inducida por el embarazo.
\end{abstract}

PALABras ClaVeS: Minidosis Acido Acetil Salicílico, Profilaxis, Hipertensión Inducida por el Embarazo.

SUMMARY: This article presents a review of the results of a low-dose Aspirin in the prevention of preeclampsia. We analyze 97 patients in three groups of risk: primigravidas, patients whit antecedent of pregnancy-induced hypertension and patients whit chronic hypertension were defined by ACOG criteria. A randomized, placebo-controlled, double-blind study was conduced between June of 1991 and October 1995 in the Hospital Militar Central (SantaFé de Bogotá, Colombia, South America). The utility of low-dose aspirin in the prevention of pregnancyinduced hypertension is not demonstrated.

KEY WORDS: Low-dose aspirin, prevention of pregnancy-induced hypertension, pregnancy induced hypertension.

\section{Introducción}

La hipertensión inducida por el embarazo (H.I.E.) es definida por el comité de terminología del Colegio Americano de Obstetricia y Ginecología como un síndrome clínico de etiología aún no aclarada, caracterizado por la coexistencia de pretensión, proteinuria y edema, el cual se presenta después de la vigésima semana del embarazo y va hasta las 48 horas del puerperio.

La incidencia de esta complicación obstétrica en diversas series realizadas en los Estados Unidos es del 7\% del total de embarazos, asociado a un $22 \%$ de muertes maternas (1). En el Hospital Militar Central la incidencia y prevalencia de la H.I.E. está entre el 3 y el $4 \%$.

La etiología de la preclampsia realmente no es conocida pero en años recientes una evidencia ha servido de soporte para la hipotesis de que el sistema de eicosanoides juega un papel importante en la producción de este cuadro clínico. Los resultados de estudios bioquímicos sugieren que existe un imbalance en la producción de estos eicosanoides vasodilatadores (la prostaciclina) y vasoconstrictores (tromboxano A2) (2).

* Médico Gineco-Obstetra, Ibagué.

** Médico Gineco-Obstetra, Dispensario Naval

*** Residente III Ginecologia y Obstetricia, Facultad de Medicina, Universidad Militar "Nueva Granada".

**** Residente II Ginecología y Obstetricia, Facultad de Medicina, Universidad Militar "Nueva Granada".
E1 tromboxano A2 es el principal producto derivado del ácido araquidónico, sintetizado por las plaquetas. Es un potente vasoconstrictor y un estimulante de la agregación plaquetaria; sus valores aumentan durante el segundo trimestre de gestaciones normales y durante el parto.

La prostaciclina es otro producto del ácido araquidónico, sintetizado en el endotelio vascular con acción vasodilatadora e inhibidora de la agregación plaquetaria; su síntesis se encuentra aumentada en el segundo trimestre del embarazo normal y disminuye al final del tercero.

Varios estudios $(3,4,5)$ han demostrado la disminución en la excreción urinaria de 6-cetoprostaglandin F1 alfa - un metabolito de la prostaciclina - en la hipertensión inducida por el embarazo, a partir del primer trimestre, con un aumento de la biosíntesis del tromboxano A2 y de su excreción urinaria.

Se cree también que el incremento de la actividad de los productos de oxidación de los radicales libres contribuiría a disminuir la síntesis de prostaciclina (2).

En ausencia de una adecuada producción de prostaciclina por el endotelio vascular, sobretodo a nivel uteroplacentario y trofoblastico la activación de las plaquetas puede ser esperada liberando tromboxano A2, serotonina y activando los mecanismos de coagulación en la microcirculacion, llevando a infartos placentarios.

Aceptando que este es el mecanismo fisiopatológico que genera la H.I.E, se podría prevenir utilizando un 
medicamento que impida la síntesis de tromboxano A2 y no inhiba la producción de prostaciclina.

Esto se podría lograr con la administración de ácido acetil salicílico a bajas dosis como lo indican numerosos estudios $(2,4,6,11)$.

El ácido acetil salicílico (A.A.S) reduce la síntesis de las prostaglandinas inhibiendo la ciclooxigenasa (acetila el residuo de alanina del sitio activo de esta) (12). Esta acción es irreversible, ya que las plaquetas - que carecen de núcleo son incapaces de resintetizar la ciclooxigenasa; necesitándose de 8 a 11 días para ser reemplazadas; y en el endotelio vascular se generara nueva ciclooxigenasa cuando el ácido acetil salicílico sea removido de la circulación.

El A.A.S., a dosis terapéuticas, reducc la formación de tromboxano A2 y de prostaciclina, pero en bajas dosis puede inhibir selectivamente la síntesis de tromboxano A2, sin afectar la producción de prostaciclina $(2,13,-17)$.

El objetivo del presente estudio consistió en evaluar si el ácido acetil salićlico ingerido a bajas dosis (100 mg./ día) a partir del segundo trimestre hasta la semana 38 de gestación prevenía la hipertensión inducida por el embarazo en las pacientes con alto riesgo de presentarla.

Con respecto a su bioseguridad durante el embarazo estudios iniciales $(18,19)$ evidenciaron una asociación con malformaciones cardíacas; pero en el proyecto colaborativo perinatal, monitorizándose 30.282 embarazos en 12 hospitales en Estados Unidos, no se comprobó esta (20), Stuart y Cols establecieron el mayor riesgo de sangrado intraparto y de aparición de petequias, hematuria $\mathrm{y}$ hemorragias subconjuntivales en el recién nacido. (21).

\section{Materiales y métodos}

Se trata de un estudio prospectivo, aleatorizado, controlado y doble ciego realizado con las pacientes usuarias del servicio de Ginecología y Obstetricia del Hospital Militar Central, entre el primero de Junio de 1991 y el primero de Diciembre de 1995.

Se estudiaron tres grupos de pacientes con alto riesgo de presentar H.I.E; primigestantes, hipertensas crónicas y con antecedentes de H.I.E en su segundo o posterior al embarazo, a las cuales se inicio aleatorimente $100 \mathrm{mg}$./ día de ácido acetil salicílico o placebo entre la duodecimosexta semana hasta la semana 38 (para evitar el aumento del sangrado intraparto y las complicaciones hemorrágicas en el recién nacido).

Los investigadores no conocían el principio activo de cada tableta, ya que ambas tenían igual presentación debido a la colaboración de los laboratorios Winthrop, y, las cajas estaban rotuladas con el numero 1 o 2 , el cual se colocaba en el protocolo de la historia clínica de la paciente para evitar un error en la administración posterior de ésta.

Cada paciente tenia su control prenatal mensual por la Unidad de Medicina Materno Fetal del servicio y entre las semanas 12 a 19 y nuevamente entre la 30 y las 34 se les solicitaron los siguientes exámenes: Glicemia - Cuadro hemático - Fibrinógeno - Reticulocitos - Plaquetas - PT PTT - Fosfatasa alcalina - Transaminasas - BUN - Creatinina - Ácido Urico - Creatinuria de 24 horas - Proteinuria de 24 horas - Depuración de creatinina y parcial de orina.
Se les realizó también una ecografía obstétrica al ingreso, a la semana 24 y a la 34 .

Para realizar el análisis del presente estudio se utilizaron las pruebas de chi cuadrado y la corrección de Yates.

\section{Resultados}

Durante el período comprendido entre Febrero de 1991 y octubre 31 de 1995 se recolectaron un total de 200 pacientes dentro del estudio de las cuales únicamente 97 terminaron el protocolo distribuidas así: prigestantes 50, con antecedentes de hipertensión inducida por el embarazo 24 e hipertensas crónicas 23.(Ver Figura. No. 1.)

Del grupo de las primigestantes (50) veintidos recibieron el medicamento I y veintiocho el medicamento II.

En el grupo de las pacientes con antecedentes de H.I.E. a 16 se les distribuyó el medicamento I y a 8 el medicamento II.

A su vez del grupo de pacientes hipertensas crónicas a 16 se les inició el medicamento I y a 7 al medicamento II (Figura. No. 2.).

De acuerdo al grupo de riesgo y al desarrollo de H.I.E. se obtuvieron los siguientes resultados:

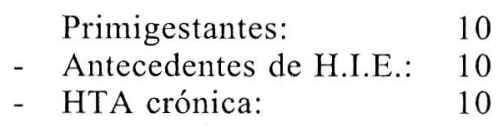

(ver Figura. No. 3)

Figura No. 1.

\section{DISTRIBUCION POR GRUPO DE RIESGO}

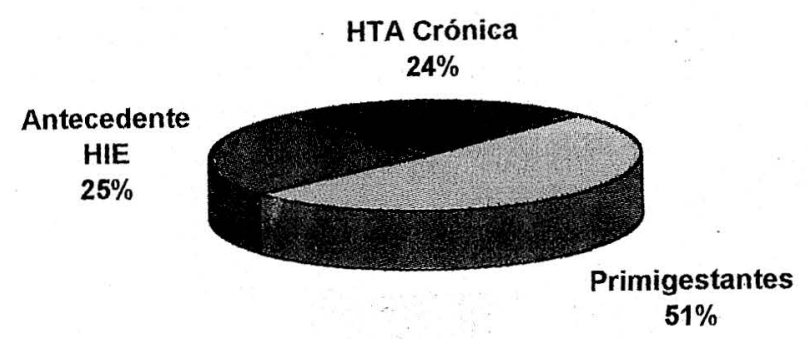

Figura No. 2.

DISTRIBUCION POR MEDICAMENTO ADMINISTRADO Y GRUPOS DE RIESGO

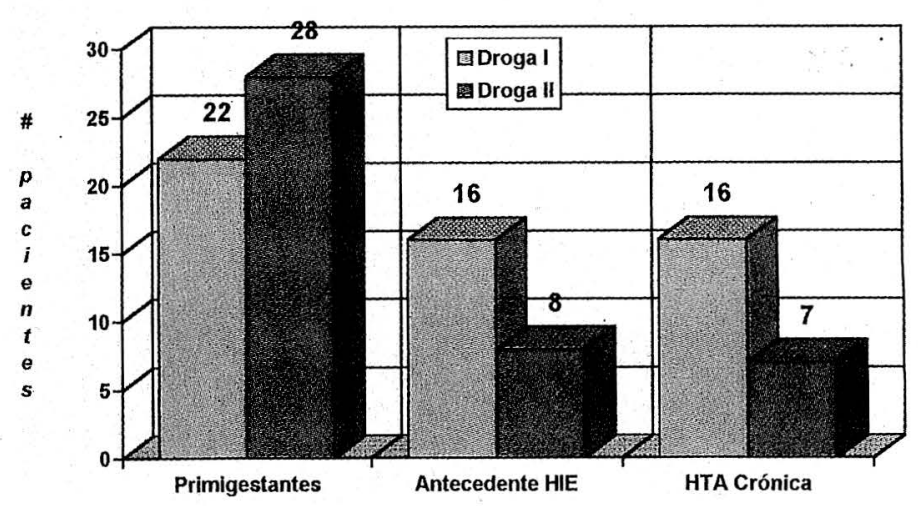


Y con respecto al desarrollo de H.I.E. en estos grupos y al consumo del medicamento I o II podemos ver:

De las diez primigestantes que desarrollaron H.I.E. cinco ingerían el medicamento I y cinco el medicamento II.

De las pacientes con antecedente de H.I.E. (10) seis ingerían el medicamento I y cuatro el medicamento II y de las hipertensas crónicas (10) ocho tomaban el medicamento I y dos el medicamento II. (Ver Figura. No. 3. )

Se revisó como objetivo importante del trabajo cada grupo de riesgo con base en el fármaco suministrado y al desarrollo de H.I.E., obteniéndose los resultados que se observan en los siguientes cuadros. (ver Figuras Nos: $4,5,6)$.

Teniendo en cuenta el tipo de H.I.E. desarrollado por las pacientes de los grupos de riesgo se encontraron los siguientes resultados:

De las pacientes primigestantes que recibieron el medicamento I, dos desarrollaron preeclampsia leve, dos hipertensión transitoria y una preeclampsia grave. Y de las que recibieron el medicamento II, dos presentaron preeclampsia leve, una preeclampsia grave y una hipertensión transitoria.

Dentro del grupo de las pacientes que tenían antecedentes de H.I.E., de las que tomaron el medicamento I cinco desarrollaron preeclampsia leve y una hipertensión transitoria; y a las que se les suministró el medicamento II, una presentó hipertensión transitoria y tres preeclampsia leve.

Figura No. 3.

DESARROLLO DE H.I.E. DE ACUERDO AL GRUPO DE RIESGO Y AL MEDICAMENTTO DADO

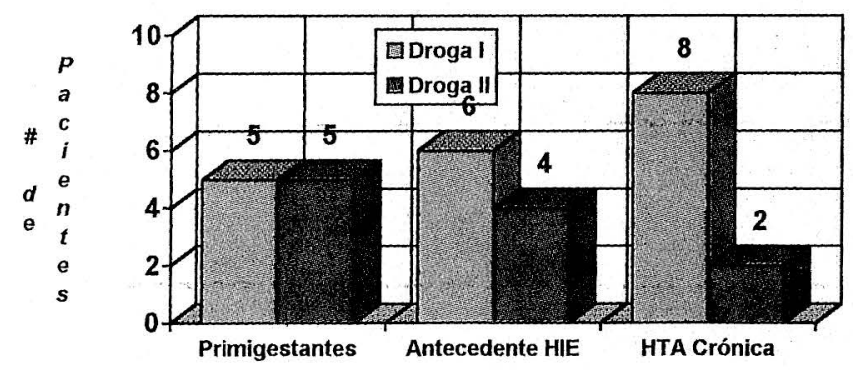

Figura No. 4.

DESARROLLO DE H.I.E. VS MEDICAMENTO DADO EN PRIMIGESTANTES

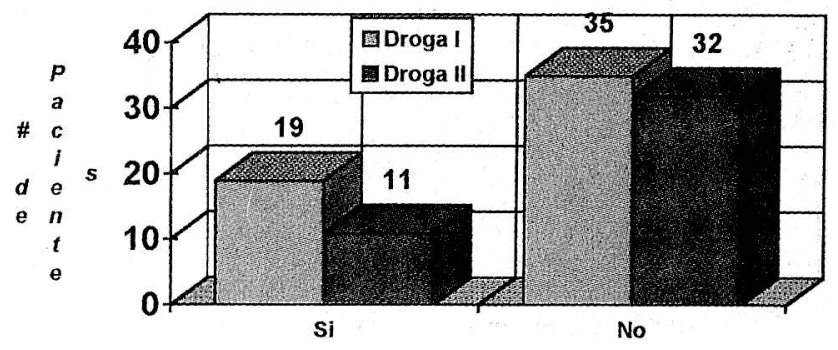

Figura No. 5.

DESARROLLO DE H.I.E. VS MEDICAMENTO DADO EN PACIENTES CON ANTECEDENTES DE H.I.E.

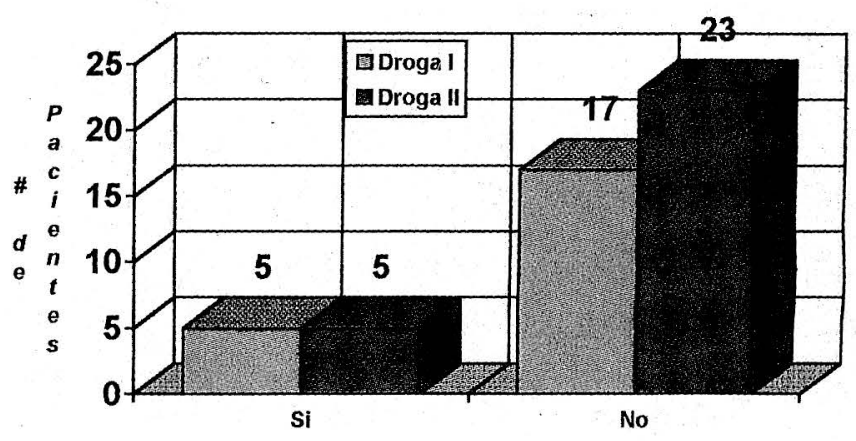

Figura No. 6.

DESARROLLO DE H.I.E. Vs MEDICAMENTO DADO EN HIPERTENSAS CRONICAS

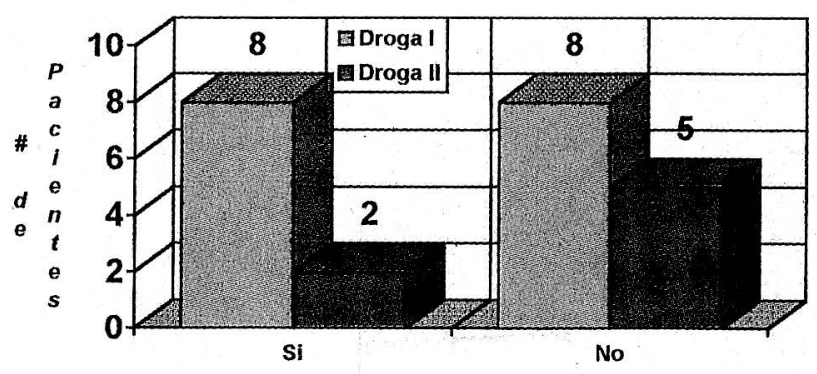

De las pacientes hipertensas crónicas que tomaban el medicamento I cinco presentaron preeclampsia grave, una preeclampsia leve, dos hipertensión transitoria; dos pacientes que recibían el medicamento II desarrollaron preeclampsia grave. (Ver figuras 7 y 8 ).

Es muy importante anotar que ninguna de nuestras pacientes presentó eclampsia, ni un síndrome HELLP, una coagulación intravascular diseminada, una ruptura hepática, ni ninguna de las otras complicaciones asociadas a la H.I.E..

\section{Plan de análisis}

Al analizar el desarrollo de H.I.E con respecto al medicamento tomado se pudo observar lo siguiente: Que no existe una diferencia significativa para el desarrollo de H.I.E. administrando el medicamento I o II, ya que el chi cuadrado obtenido es menor que el valor teórico de éste.

Con respecto al grupo de primigestantes y el desarro110 de H.I.E. se obtiene una diferencia estadística significativa entre la aparición de H.I.E y la administración del medicamento I o II.

En las pacientes con antecedentes de H.I.E., y las hipertensas crónicas tampoco se obtuvieron diferencias estadísticas significativas, al comparar el desarrollo o no de H.I.E. con el medicamento suministrado. 
Figura No. 7.

TIPO DE H.I.E. DE ACUERDO AL GRUPO DE RIESGO

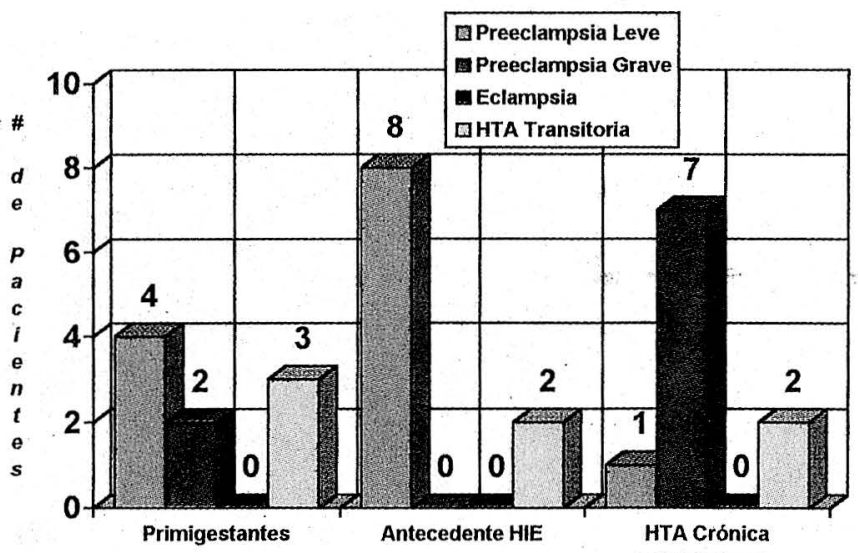

Figura No. 8 .

TIPO DE H.I.E. DE ACUERDO AL MEDICAMENTO INGERIDO

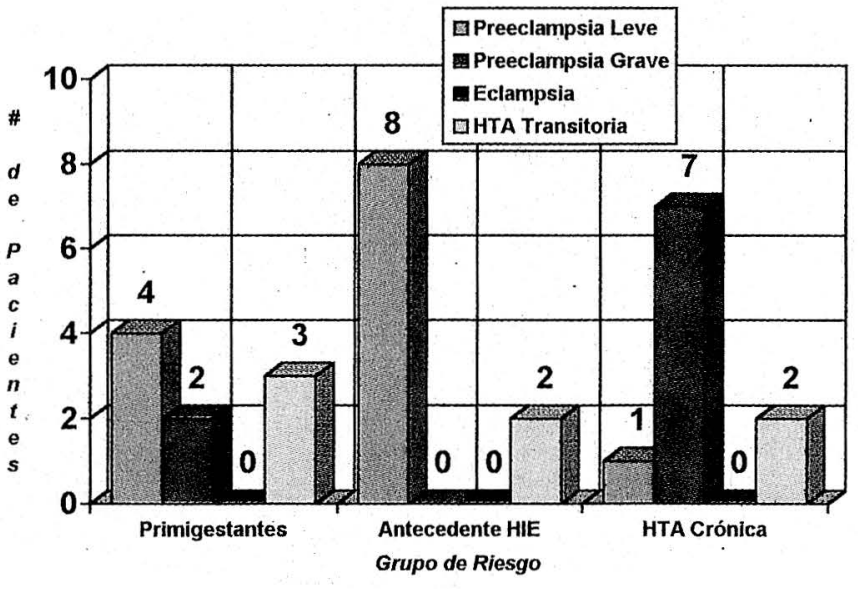

Al relacionar el tipo de H.I.E. con el medicamento ingerido, se puede inferir también que no hay diferencia estadísticamente significativa entre la aparición de algún tipo de H.I.E. y la ingestión del fármaco I o II.

\section{Discusión}

Desde 1979, año en que Isherwoord y Crandon (3) demostraron que las mujeres primigestantes que ingerían A.A.S, al menos una vez cada 2 semanas tenían menos riesgo de desarrollar hipertensión durante el embarazo, se han realizado muchos estudios $(6,10,11,20$, ) para tratar de comprobar dicha hipótesis y evaluar sus efectos secundarios.

En 1991, Imperiale y Cols. publicaron un análisis que comparaba los estudios realizados por Bequhls en 1985, Wallemburg en 1986, Wallemburg y Rotmans en 1987, Benihn en 1989, Schiff en 1989 y Mc Parlond en 1990 y concluía que los bajas dosis de A.A.S., reducían el riesgo de HIE y de RCIU severo, sin presentar efectos adversos materno-neonatales.

En el presente trabajo no encontramos evidencia significativa para determinar que grupo de riesgo tiene mayor tendencia a desarrollar H.I.E., independientemente del fármaco administrado. No se presentaron complicaciones mayores de la H.I.E. como la eclampsia, síndrome HELLP, CID, ruptura hepática. Aunque se ha dicho que con la ingestión de A.A.S., existe la posibilidad de la prolongación del embarazo, durante el presente estudio no hubo ningún embarazo postérmino. No se observaron diferencias estadísticamente significativas en la vía del parto y la ingestión del fármaco I o II.

\section{Agradecimientos}

Al departamento de Ginecología y Obstetricia del Hospital Militar Central por su colaboración en la remisión y control de las pacientes.

A los laboratorios Sanofi, Winthrop, por la elaboración y presentación de los 2 medicamentos A.A.S y placebo).

\section{BIBLIOGRAFIA}

1. Arias F. Practical Guide to high-risk pregnancy and delivery. Chapter 10. Second edition St. Louis Mosby Year Book, 1993, p. 183-210.

2. Dekker GA and Sibay BM. Low-dose aspirin in the prevention of preeclampsia and fetal growth retardation: Rationale, mechanisms, and clinical trials. PDNA.

3. Fitzgerald DJ., Rocky W., Murray R., Mayo G and Fitzgerald, G. Thromboxane A2 synthesis in pregnancy-induced hypertension. Lancet 1990; 335: 751-754.

4. Ylikorkala $O$ and Makila M.Prostacyclin and thromboxane in gynecology and obstetrics. Am J Obstet Gynecol 1985 152: 318-329.

5. Burrow G., Ferris T. Medical Complications during pregnancy. Chapter one, fourth Edition. Philadelphia WB scunders company 1995, p: 1-28.

6. Benigni A., Gregorini G., Frusca T., Chiapiccinelli A., Pinciroli V., Fanelli R., Castaldi A., Remuzzi G. Effects of low-dose aspirin on fetal and maternal generation of thromboxane by platelets in women at risk for pregnancy-induced hypertension. N Engl J Med 1989; 321 : 357-362.
7. Sibai BM., Mirra R., Chesney CM and Leffler C. Low-dose aspirin in pregnancy. Obstet Gynecol 1989; 74: 551-556.

8. Thorp JA., Walsh SW and Brath PC. Low-dose aspirin inhibits thromboxane, but not prostacyclin, production by human placental arteries. Am J Obstet Gynecol 1988; 159: 1381-1384.

9. Wallemburg H. CS., Dekker GA., Makovitz JW and Rotmans P. Lowdose aspirin prevents pregnancy-induced hypertension and preeclampsia in angiotensin-sensitive primigravidae. Lancet 1986; 1:1-3.

10. Wallemburg H. CS., Dekker GA., Makovitz JW and Rotmans N. Effect of low-dose aspirin on vascular refractoriness in angiotensinsensitive primigravid women. Am J Obstet Gynecol 1991; 164: 1169-1173.

11. Sánchez-Ramos L., O’Sullivan MJ., and Garrido-Calderón J. Effect of low dose aspirin on angiotensin II pressor response in human pregnancy. Am J Obstet Gynecol 1987; 156: 193-194.

12. Goodman A., Goodman L., and Gilman A. Farmacológicas de la terapéutica. Capítulo 29. Sexta edición. Buenos Aires, Editorial Médica Panamericana, 1982 p. 657-687.

13. Calero R., Rodríguez R. El sindrome de preclampsia-eclampsia. Revisión del Servicio de Toxemias del Instituto Materno Infantil. 
14. Vlinikka L, Hartikainen S, y Licorkala O. Low dose aspirin in hypertensive pregnant women: effect on pregnancy out come and prostacyclin-thromboxane balance in mother and newborn. $\mathrm{Br} \mathrm{J}$ Obstet Gynecol. 1993; 100 (9): 809-15.20. Rudolph AM.Effects of aspirin and acetaminophen in pregnancy and in the newborn. Arch Intern Med 1981; 141: 358-363.

15. Remuzzi G., Ruggenenti P. Prevention and treatment of pregnancyassociated hypertension: what have we learned in the last years? Am J Kidney Dis. 1991; 18 (3): 285-305.

16. Lindheimer., Katz: Preclampsia; Pathophisiology, diagnosis, and management. Annu Rev Med; 1989; 48: 233-250.

17. Walsh. Low dose aspirin: Treatment for the imbalance of increased thromboxane and decreased prostacyclin in preeclampsia. Am J Perinatol; 1989; 6 (2): 124-32.21. Corby DG. Aspirin in pregnancy: Maternal and fetal effects. Pediatrics 1978; 62 (Suppl): 930-937.

18. Rudolph AM. Effects of aspirin and acetaminophen in pregnancy and in the newborn. Aich Intern Med 1981; 141: 358-363.
19. Corby DG. Aspirin in pregnancy: Maternal and fetal effects. Pediatrics 1978; 62 (Suppl): 930-937.

20. Slone D., Siskind V., Heinanen OP., Monson RR., Kaufman DW and Shapiro S. Aspirin and congenital malformations. Lancet 1976; 1 : 1373-1375.

21 Stuard J., Grass SJ., Elrad H., and Graeber JE. Effects of acetysalicylicacid ingestion on maternal and neonatal hemostasis. N Engl J Med 1982; 307:909-912.

22. Sibai BM., Carritis SN. Prevention of preeclampsia with low-dose aspirin in healthy, nulliparous pregnant women. $\mathrm{N}$ England $\mathrm{J}$ med; 1993; 329 (17): 1213-1218.

23. Hauth JC., Goldenberg RL.,Parker CR. Low dose aspirin therapy to prevent preeclampsia. Am J Obstet Gynecol; 1993; 168 (4): 10831091.

:4. Schrocksnadel H., Sitte B., Alge A. Low dose aspirin in primigravidae with positive roll over test. Gynecol Obstet Invest; 1992; 34 (3): 146-150. 